\title{
A fatal outcome of thoracic aortic aneurysm in a male patient with bicuspid aortic valve
}

\author{
Blanka Milanowska1 ${ }^{1}$ Ewa Michalak1, Ilona Michałowska², Eugeniusz Szpakowski , Anna Konopka, \\ Anna Klisiewicz ${ }^{5}$ Zofia Teresa Bilińska ${ }^{1}$
}

1 Unit for Screening Studies in Inherited Cardiovascular Diseases, Institute of Cardiology, Warsaw, Poland

2Department of Radiology, Institute of Cardiology, Warsaw, Poland

${ }^{3}$ Department of Cardiac Surgery and Transplantation, Institute of Cardiology, Warsaw, Poland

${ }^{4}$ Intensive Cardiac Therapy Clinic, Institute of Cardiology, Warsaw, Poland

${ }^{5}$ Department of Adult Congenital Heart Diseases, Institute of Cardiology, Warsaw, Poland

Postep Kardiol Inter 2013; 9, 3 (33): 265-271

DOI: $10.5114 /$ pwki.2013.37507

\begin{abstract}
Thoracic aortic aneurysm is often an asymptomatic but potentially lethal disease if its most catastrophic complication - aortic dissection - occurs. Thoracic aortic dissection is associated with a high mortality rate despite ongoing improvement in its management. We report a fatal outcome of thoracic aortic aneurysm in a male patient with bicuspid aortic valve. The patient was qualified for elective surgery of the ascending aorta and aortic valve at the age of 39 but he did not agree to undergo the proposed procedure. Three years later, he experienced acute aortic dissection and died despite a prompt diagnosis and complex management.
\end{abstract}

Key words: thoracic aortic aneurysm, acute aortic dissection.

\section{Introduction}

Bicuspid aortic valve (BAV) is one of the most common congenital heart diseases, found in 1-2\% of the population [1]. Bicuspid aortic valves are prone to either aortic valve regurgitation or aortic valve stenosis and many BAV patients require aortic valve surgery during their lives. However, BAV disease is not only a disorder of valvulogenesis; it probably represents a more global disorder of cardiac and vascular morphogenesis. Nonvalvular findings have been reported in up to $50 \%$ of BAV adults, including coarctation of the aorta, hypoplastic left heart syndrome, ventricular septal defect, patent ductus arteriosus and atrial septal defect. The most common abnormality associated with BAV is dilatation of the thoracic aorta [2, 3]. Svensson et al. showed that $20 \%$ of patients who underwent BAV surgery had a concomitant ascending aortic aneurysm that required surgery [4]; other reports show even higher (50-60\%) prevalence of aortic dilatation in BAV patients $[5,6]$.

\section{Case report}

A 39-year-old male patient with a history of untreated arterial hypertension was admitted to the Institute of Cardiology for the first time in 2007 due to suspected acute coronary syndrome (ACS), as he suffered from atypical chest pain. Physical examination revealed blood pressure 180/110 mm Hg, heart rate of 74 beats per minute, loud systolic murmur at the base of the heart, and no symptoms of heart failure. ECG showed sinus rhythm 73 beats per minute, features of left ventricle hypertrophy (LVH) and negative $\mathrm{T}$ waves in II, III, aVF. Coronary angiography was performed immediately and it revealed atherosclerotic lesions without occlusive coronary artery disease (CAD): maximum luminal diameter stenosis was $30-40 \%$ in the right coronary artery (RCA). The troponin I level was detectable but not significantly elevated $(0.03 \mathrm{ng} / \mathrm{ml}$ and subsequently $0.07 \mathrm{ng} / \mathrm{ml}$, normal values $<0.10 \mathrm{ng} / \mathrm{ml})$ and the D-dimer level was elevated $(1.0 \mu \mathrm{g} / \mathrm{ml}$, normal values $<0.5 \mu \mathrm{g} / \mathrm{ml})$.

\section{Corresponding author:}

Blanka Anna Milanowska MD, Unit for Screening Studies in Inherited Cardiovascular Diseases, Institute of Cardiology, 42 Alpejska St, 04-628 Warsaw, Poland, tel.: +48 722227 166, e-mail: b.milanowska@ikard.pl

Received: 30.06.2013, accepted: 12.07.2013. 


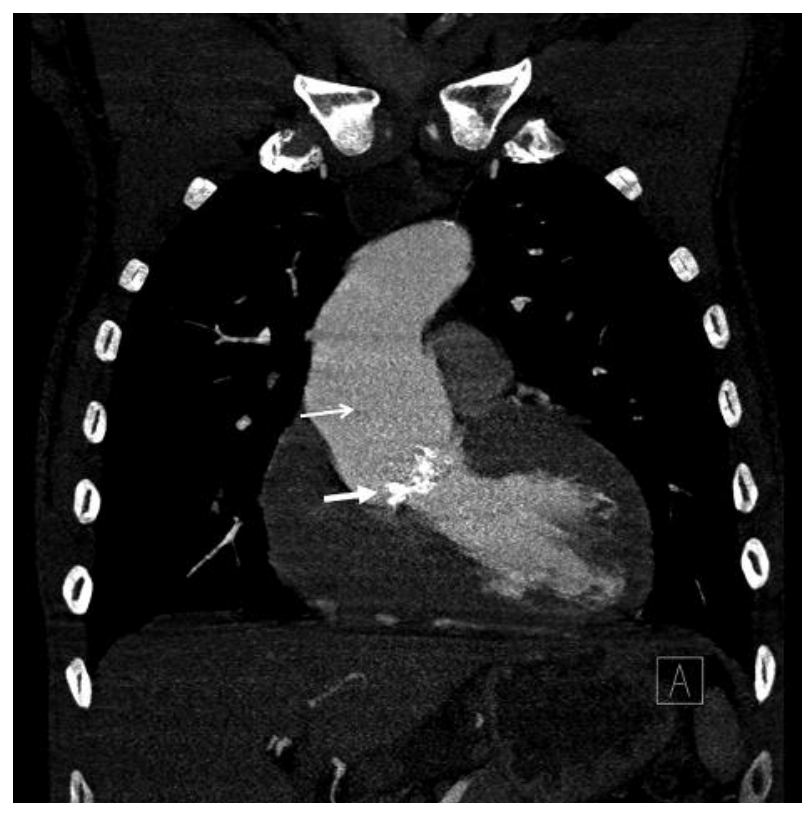

Fig. 1. ECG-gated angio-CT of the thoracic aorta, MPR (multiplanar reformatted) coronal image shows an aneurysm of the ascending aorta (thin arrow), severe calcification of the aortic valve (thick arrow)

As ACS was excluded, further tests were performed. Echocardiography revealed BAV with degenerative changes and calcifications, aortic stenosis with the mean pressure gradient of $45 \mathrm{~mm} \mathrm{Hg}$, mild aortic regurgitation, disproportionate concentric left ventricle hypertrophy with maximum wall thickness up to $1.7 \mathrm{~cm}$ and normal left ventricle systolic function (LVEF 65\%). Ascending aorta diameter when measured in end-diastole was $5.6 \mathrm{~cm}\left(2.6 \mathrm{~cm} / \mathrm{m}^{2}\right.$ when indexed for body surface area (BSA) and Z-score including both BSA and age 9.23), thus meeting the diagnostic criteria for thoracic aortic aneurysm (TAA). Computed tomography (CT) confirmed BAV and dilatation of the ascending aorta up to $5.5 \mathrm{~cm}$; aortic dissection (AD) was not discovered (Figure 1). Other comorbidities were chronic pancreatitis with recurrent exacerbations, probably due to alcohol overuse, type $C$ chronic viral hepatitis and hyperlipidemia. As the patient met the operating criteria for TAA, he was offered cardiothoracic surgery. Yet, he refused to undergo the surgery and he did not change his mind for the following 3 years. The patient received pharmacological treatment including $\beta$-blocker, angiotensin-converting enzyme inhibitor (ACEI), diuretic, atorvastatin, and acetylsalicylic acid (ASA). It is not known how the blood pressure was controlled during the following 3 years because of the patient's poor compliance.

In January 2010, the patient experienced a cerebrovascular event (aphasia of sudden onset that lasted for more than $24 \mathrm{~h}$ ). During the hospitalization in a Department of Neurology, echocardiography revealed aortic valve morphology and aorta dimensions comparable with those of the previous examinations. The consulting physician performed ultrasonography of cervical arteries; they were calcified but not dissected.

Three months later, in April 2010, the patient was admitted to the emergency room in our institution due to an abrupt onset of chest pain that persisted for the following $3 \mathrm{~h}$. At admission, the patient suffered from severe retrosternal pain that radiated to the left upper limb. The physical examination showed a loud systolic murmur at the base
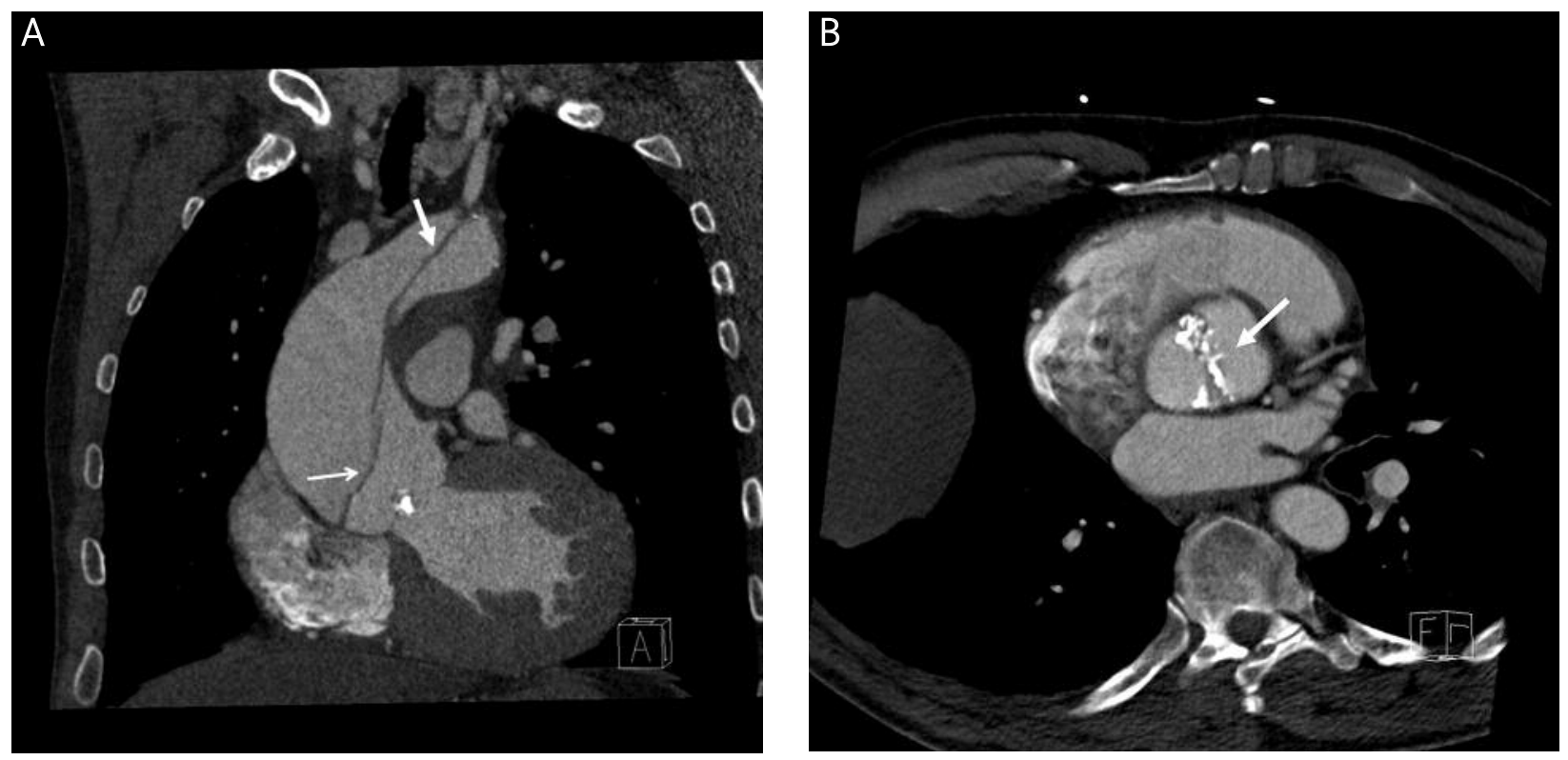

Fig. 2. ECG-gated angio-CT of the thoracic aorta. A - MPR coronal image shows dissection of the ascending aorta (thin arrow) and aortic arch (thick arrow). B - MPR reconstruction parallel to aortic valve reveals the BAV (bicuspid aortic valve) with severe calcifications (arrow) 
of the heart, blood pressure was $163 / 98 \mathrm{~mm} \mathrm{Hg}$ in the right arm, and $135 / 93 \mathrm{~mm} \mathrm{Hg}$ in the left arm, heart rate of 65 beats per minute. Twelve-lead standard ECG showed sinus rhythm 72/min, LVH features with negative T waves in II, III, aVF and V3-V6 - negative T waves in anterolateral leads were new findings in comparison to the previous ECG. Troponin I level at admission was mildly elevated $0.34 \mathrm{ng} / \mathrm{ml}$ (normal values $<0.10 \mathrm{ng} / \mathrm{ml}$ ).

The patient's medical history (BAV, TAA, arterial hypertension, coronary angiography performed 3 years earlier without hemodynamically significant atherosclerotic plaques) combined with clinical presentation (severe chest pain and blood pressure difference between upper limbs) made acute aortic dissection (AAD) the most probable diagnosis. Bedside transthoracic echocardiography (TTE) performed immediately revealed a dissection flap in the dilated ascending aorta. The diagnosis was confirmed with CT (Figures 2 and 3 ). It revealed further dilatation of the ascending aorta up to $6.3 \mathrm{~cm}$ and aortic dissection with intimal tear localized about $2.5 \mathrm{~cm}$ above the aortic valve; the dissection flap extended from the right coronary artery ostium to the distal part of the aortic arch. The dissection comprised the right subclavian artery, which was found to be aberrant, the right common carotid artery and the proximal segment of the left subclavian artery.

The patient received typical pharmacological treatment and was immediately referred to undergo surgery. The Bentall procedure - total aortic root replacement with St Jude Medical $29 \mathrm{~mm}$ conduit of the aortic valve, aortic root and ascending aorta with re-implantation of the coronary arteries into the graft - was performed. The distal part of the ascending aorta was replaced with a Datascope $22 \mathrm{~mm}$ prosthesis. The course of the early period following the operation was typical: the patient was conscious, respiratory sufficient, circulation was briefly supported with dobutamine, and LVEF assessed by TTE was 50\%. He was extubated on the $1^{\text {st }}$ postoperative day and for the next 2 days he was in a stable condition. On the $4^{\text {th }}$ postoperative day he started to be excited and restless; fever of $39^{\circ} \mathrm{C}$ appeared. From the $6^{\text {th }}$ postoperative day, the patient started to develop symptoms of septic shock and multiorgan failure. The mediastinum was drained and empiric wide-spectrum antibiotic therapy was administered. Despite intensive care treatment, the patient died on the $8^{\text {th }}$ day after surgery due to cardiopulmonary insufficiency.

\section{Discussion}

\section{Clinical presentation}

Current American Heart Association (AHA) guidelines on thoracic aortic diseases specify high-risk conditions for aortic dissection [7]. They include conditions associated with an increased aortic wall stress (hypertension, weight lifting or other Valsalva maneuver, trauma, cocaine or other stimulant intake) and conditions associated with aortic wall abnormalities (such genetic abnormalities as Marfan syndrome (MFS), Loeys-Dietz syndrome (LDS), BAV including prior aortic valve replacement (AVR); and nongenetic abnormalities such as inflammatory vasculitis, pregnancy, and corticosteroids administration). About $70 \%$ of patients with AD have a history of hypertension, which is often uncontrolled [8]. Genetically based and congenital diseases are important factors predisposing to $A D$ in the younger population. Known genetic defects associated with TAA affect mostly the aortic root and they typically
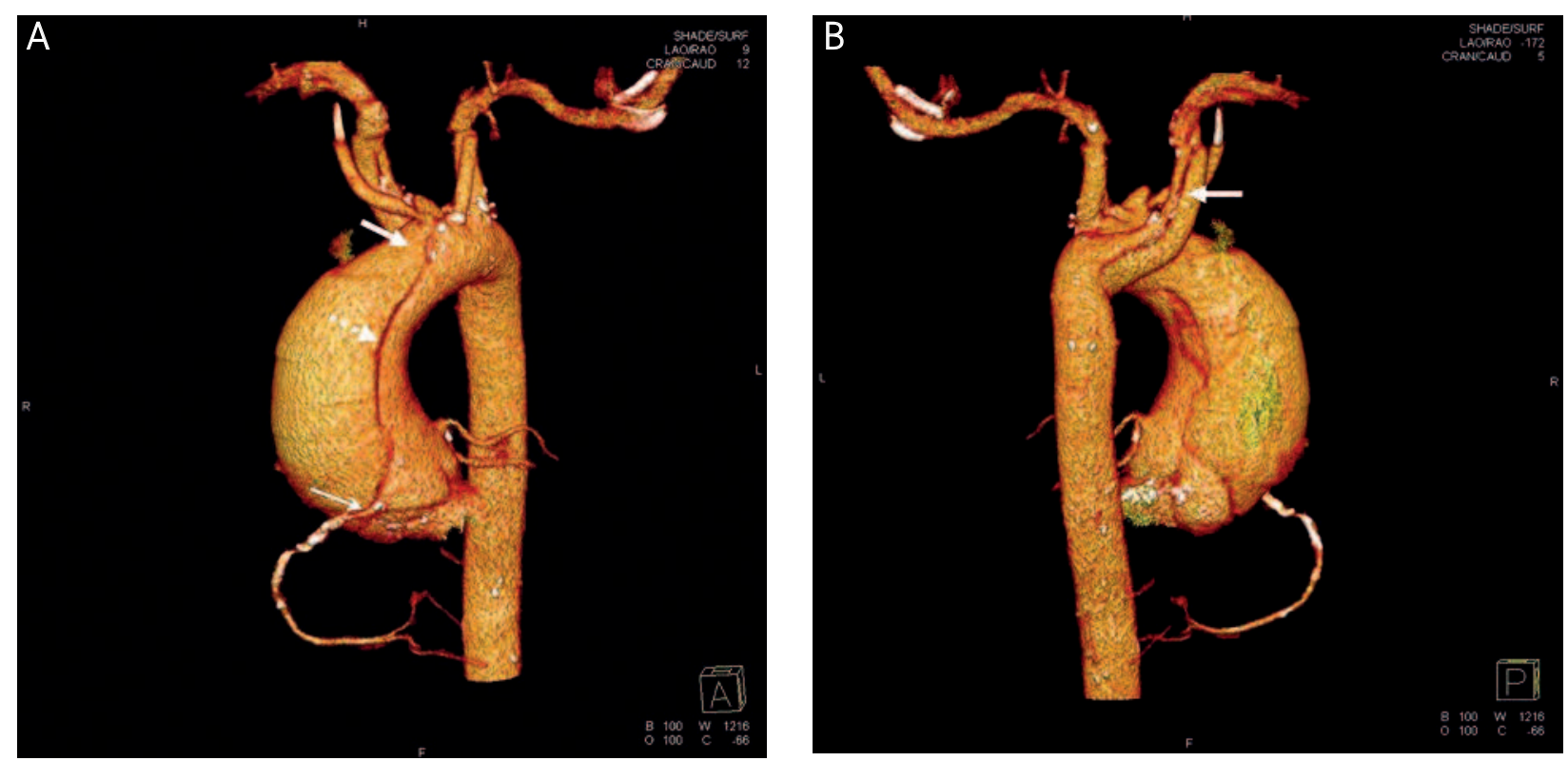

Fig. 3. ECG-gated angio-CT of the thoracic aorta, 3D-VR (volume rendering). A - Images show type A dissection of the aneurysmal aorta. Anterior view: a dissection of the aorta (broken arrow) extending from the right coronary artery ostium (thin arrow) to the distal part of the aortic arch (thick arrow). B - Posterior view: aberrant right subclavian artery, which is also dissected (arrow) 
lead to type A dissection. According to the International Registry of Aortic Dissection (IRAD study) $50 \%$ of patients younger than 40 years of age had MFS [9]. Bicuspid aortic valve is present in about $15 \%$ of patients with type A AD. The 2010 AHA guidelines for the first time stressed the significance of familial history as an important risk factor for AD [7]. At least $20 \%$ of patients with AD without known genetic syndromes have relatives with aTAA or dissection [10]. An obvious risk factor for $A D$ is dilatation of the aorta. It is still the best marker of $A D$ and the parameter widely used to qualify patients for prophylactic aortic surgery.

Dilatation of the aorta in BAV patients may be independent of the functional competence of BAV [11-13], as the thoracic aorta in those patients may undergo accelerated degenerative changes [14]. Decreased amount of fibrillin, elastin fragmentation and apoptosis of smooth muscle cells in the thoracic aorta of BAV patients have been described. Deficient fibrillin-1 results in smooth muscle cell detachment, matrix disruption and cell death. Matrix metalloproteinases could be involved in this process [15-18].

Our patient presented some of the conditions predisposing to AD: TAA, BAV and arterial hypertension. He did not present any signs of MFS or any other genetic syndrome; his family history was negative, too.

According to the IRAD study, the most common symptom of acute aortic dissection is pain (95\%), most often chest pain $(73 \%)$, which is abrupt in its onset ( $85 \%)$, severe in intensity, described as sharp more often than ripping or tearing. It may change its location due to further extension of the dissection process along the aortic wall. IRAD data showed that patients with type A dissection typically present with chest pain (79\%), more commonly described as anterior (71\%), while patients with type B dissection frequently present with back pain (64\%). Patients with type A dissection may also report back pain (47\%) and abdominal pain (21\%) as a result of extension of the dissection into the descending aorta. Other manifestations of AD are: syncope (9.4\%), stroke $(4.7 \%)$ or congestive heart failure $(6.6 \%)$, limb, renal or mesenteric ischemia, superior vena cava syndrome, Horner syndrome, hemoptysis and cough [8]. The variability of clinical manifestations is wide ranging from a typical intensive pain to painless discrete signs and symptoms.

A physical examination may reveal murmur of aortic insufficiency, pulse deficit, symptoms of heart failure or a cerebrovascular event. Murmur of acute aortic insufficiency described in one third of patients with $A D$ ( $44 \%$ in type $A$ ) is particularly significant if it is a new finding. Pulse deficit, if present, is strongly suggestive of $A D$; however, it is present in no more than $31 \%$ of patients. Half of the patients have systolic blood pressure $150 \mathrm{~mm} \mathrm{Hg}$ or higher at the initial examination, but they may also be normotensive, hypotensive or even present symptoms of shock. None of the findings is specific for AD; moreover, patients often do not present any of them [8, 19]. As classic physical findings are often absent, the diagnosis of $A D$ requires a high index of clinical suspicion.
Rogers et al. analyzed the sensitivity of risk factors (including conditions predisposing to $A D$, clinical symptoms and examination findings) proposed in the 2010 AHA guidelines. The most common high-risk predisposing conditions identified in their study were known TAA (14.7\%) and known aortic valve disease (11.9\%), whereas the most common highrisk examination findings included a new murmur of aortic insufficiency accompanied by pain (23.6\%) and a pulse deficit or systolic blood pressure difference between extremities (20.3\%). They proposed a detection risk algorithm for the identification of $A D$ at initial diagnosis, which was highly sensitive (98\%) [20]. As they verified their score only in the IRAD population (patients with confirmed AD), its specificity is not known. Further studies are needed to elaborate a validated and clinically useful risk score. As long as it does not exist, it is important to keep risk factors for $A D$ in mind. Data show that $A D$ is suspected at initial evaluation in fewer than half of the patients ultimately diagnosed with the disease $[8,21,22]$. The diagnosis is often delayed for several hours. The time from initial symptoms to hospital admission is approximately $1-2 \mathrm{~h}$, as many of the AAD patients have symptoms making them search for medical support. Yet, in $50 \%$ of patients with type A dissection the time elapsed from initial symptoms to diagnosis is $>6 \mathrm{~h}$ in Europe and $>15 \mathrm{~h}$ in the U.S.A. [23].

Our patient experienced a fairly typical clinical manifestation with severe abrupt anterior chest pain, which may indicate that the site of initial intimal disruption is localized in the ascending aorta. He presented a blood pressure difference between the upper limbs and systolic murmur of the aortic valve stenosis. A diastolic murmur of the aortic valve due to its acute incompetence, typically described for AD, was not observed. Significant aortic regurgitation (moderate or severe) is less likely in the presence of huge calcifications of the aortic valve described previously in the patient presented.

\section{Differential diagnoses}

Differential diagnoses of AAD include ACS with or without ST segment elevation, pulmonary embolism, pericarditis, pleuritis, musculoskeletal pain, aortic aneurysm without dissection or aortic stenosis.

Acute coronary syndrome is the most common cause of life-threatening chest pain and also the most common cause of AAD misdiagnosis [24]. However, some differences in its clinical presentation have been described. The pain associated with ACS starts slowly and gains in intensity with time; it is usually oppressive and dull, while the pain that AAD patients typically present with is abrupt, very intense, with its maximum at the time of onset. Such physical findings as blood pressure difference between upper limbs are not part of the ACS symptoms. Moreover, since the patient had coronary arteriography performed 3 years earlier, which did not show any hemodynamically significant coronary artery lesions, the probability of ACS as the only cause of chest pain was lower. However, it is important to remember that 20\% of $A D$ patients have concomitant coronary ischemia as $A D$ 
may extend into the coronary ostium, usually the one of the right artery [8].

Known factors predisposing to pulmonary embolism (PE) include hip or leg fracture, surgery (particularly orthopedic, but also other major procedures), prolonged immobility due to a disease or trauma, malignancy or thrombophilia. Clinical manifestations of PE may vary; however, most patients have at least one of the following symptoms: dyspnea, chest pain (usually pleuritic) or syncope [25]. As far as the reported patient is concerned, he presented blood pressure difference between the limbs strongly suggestive of $A D$ but not typical of PE.

\section{Imaging modalities}

Different imaging modalities can be employed to confirm AD diagnosis: TTE, transesophageal echocardiography (TEE), CT, magnetic resonance imaging (MRI), aortography, and intravascular ultrasound (IVUS).

Transthoracic echocardiography is a noninvasive, widely available technique, which can be used for decision making in the emergency room. For AD detection, its sensitivity is 59$83 \%$ and specificity $63-93 \%$. The sensitivity of TTE for the diagnosis of type A dissection ranges from $78 \%$ to $100 \%$, but it is only $31-55 \%$ for type B dissection, as the descending aorta is not easily visualized [26]. Transesophageal echocardiography has a sensitivity of $94-100 \%$ and a specificity of $77-$ $100 \%$ for the diagnosis AD $[27,28]$. A disadvantage of this method is its semi-invasive nature and insufficient evaluation of the distal part of the ascending aorta and the branches of the aortic arch [29]. The technique requires skilled staff to perform it and to interpret its findings.

Multidetector CT is often the modality of choice for the evaluation of AD. It is a widely available, fast and accurate method. Helical CT has $100 \%$ sensitivity and $98 \%$ specificity $[27,30,31]$. The accuracy of modern multidetector CT that allows rapid image acquisition and data reconstruction is almost $100 \%$ [32]. The diagnosis is based on the detection of an intimal flap which separates two lumens: the true one and the false one. Computed tomography allows type A dissection to be differentiated from type $B$, and it can localize the intimal tears and assess aortic branch-vessel involvement.

A relatively new modality is "triple-rule out" $\mathrm{CT}$. The term means ECG-gated multidetector CT angiography performed in an emergency department as a screening study to evaluate patients with acute chest pain for three potential fatal causes: $A D, P E$, and $C A D$, with high negative predictive value (>95\%) [33-35].

Magnetic resonance imaging is a noninvasive method, both highly sensitive and specific (nearly 100\%), for diagnosing AD. Contrast-enhanced MR angiography has several advantages over CT angiography, including lack of nonionizing radiation and greater vessel coverage at high resolution with fewer slices. Magnetic resonsnce is not widely performed for the diagnosis of $A A D$ due to limited availability and long time of acquisition, so it cannot be performed in hemodynamically unstable patients [27].

Aortography, considered as the diagnostic standard for $A D$ for several decades, is nowadays rarely performed as modern non-invasive methods have developed. It is highly specific (better than 95\%) for diagnosis of classical AD, but its sensitivity is lower in cases of atypical forms of AD (intramural hematoma $(\mathrm{IMH})$, penetrating aortic ulcer (PAU)) $[36,37]$.

Intravascular ultrasound, with its sensitivity and specificity close to $100 \%$ in evaluating $A D$, overcomes known limitations of conventional aortography as the aortic wall is visualized from inside the lumen. It is particularly useful in assessing the presence of IMH or PAU if other methods give inadequate results, but as an invasive procedure and due to its limited availability, it is not a first step modality in $\mathrm{AD}$ diagnosis $[37,38]$.

The 2010 AHA guidelines recommend urgent and definitive imaging of the aorta using TEE, CT or MRI to identify or exclude thoracic aortic dissection in patients at high risk for the disease (IB) [7]. The aim is to confirm the diagnosis, differentiate true and false lumens, localize intimal tears and dissection extent, distinguish communicating and non-communicating dissections, assess branch involvement, detect and grade aortic regurgitation and detect extravasation. In the IRAD study, most patients underwent multiple imaging studies. The first diagnostic test was most often CT (61\%), followed by echocardiography (33\%), MRI (2\%) and angiography (4\%). Intravascular ultrasound, as a fairly new technique, was not evaluated in the IRAD study [8]. A comparative study with spiral CT, 0.5 Tesla MR and TEE showed $100 \%$ sensitivity for all techniques, with better specificity of CT (100\%) than of TEE (94\%) and MR (94\%) [31]. Another recent meta-analysis that evaluated the diagnostic value of TEE, helical CT and MR for suspected AD found that all of them provided equally high diagnostic accuracy [27]. Selecting a specific diagnostic technique should be based mostly on the following factors: immediate availability of the method, experience of the imaging staff and patient variables (hemodynamic state or specific contraindications).

ECG should be obtained from all patients who present symptoms suggesting of AD [7]. Up to $30 \%$ of patients with type A AD have normal ECG; the others may have non-specific ST-segment or T-wave changes (42\%), LVH features (25\%), signs of ischemia (17\%), Q waves (7.7\%), or new myocardial infarction signs including ST segment elevation (4.8\%) [8]. If the risk for $A D$ is high, the diagnostic procedure for $A D$ should be continued independently of ECG findings.

Highly specific and sensitive laboratory tests for acute aortic diseases are not available yet. However, some biochemical markers seem to be very promising. D-dimer a biodegradation product of fibrin - has almost $100 \%$ sensitivity in diagnosing AAD; its serum concentrations reflect the longitudinal extent of the dissection and correlate with mortality rates. However, as a nonspecific indicator of 
intravascular coagulation, D-dimer can be elevated in many conditions. It seems to be a useful marker to identify patients who do not have AD. The marker has an excellent negative predictive value of $95 \%$ within the first $24 \mathrm{~h}$ of onset at the widely used cutoff level of $500 \mathrm{ng} / \mathrm{ml}$. Several other plasma markers have been investigated for their utility in the evaluation of AAD. Plasma smooth muscle myosin heavy chain protein, high-sensitivity C-reactive protein and elastin degradation products have shown some diagnostic value, but we need further prospective studies to elaborate recommendations regarding their use [39-42].

\section{Treatment options}

A patient with suspected or confirmed diagnosis of AAD should be treated in an intensive care unit. Initial management including $\beta$-blockers or vasodilators is directed at decreasing the aortic wall stress by controlling heart rate and blood pressure. The aim is to obtain a heart rate below 60 beats per minute and systolic blood pressure between $100 \mathrm{~mm} \mathrm{Hg}$ and $120 \mathrm{~mm} \mathrm{Hg}$ [7, 37].

The definitive management depends on the type of $A D$. A patient with dissection involving the ascending aorta (type A AD) should be immediately evaluated for surgical repair to prevent life-threatening complications including aortic rupture or cardiac tamponade [7, 37]. The mortality in untreated type A dissection is extremely high: $1 \%$ per hour with half of the patients dead after $48 \mathrm{~h}$. After 1 month and 1 year only $16 \%$ and $8 \%$ of patients respectively are still alive [43].

The technique for repairing type $A A D$ depends on the size of the aortic root, the condition of the aortic valve, extent of the dissection and anatomical conditions. The goals of surgery are to resect the primary entry tear, to correct or prevent any malperfusion and aortic valve regurgitation and, if possible, to prevent late complications in the proximal and downstream aorta. There is a large variability of surgical procedures including valve preserving surgery - tubular graft implantation if the aortic root and the aortic valve are unchanged, Bentall procedure - total aortic root replacement with a composite valve graft consisting of the aortic root and a prosthetic valve and reimplantation of the coronary arteries into the graft and finally a technically difficult David procedure, involving ascending aorta replacement with reimplantation of the native valve into the aortic graft and attachment of the graft to the left ventricle outflow tract [7, 37].

\section{Conclusions}

The case report shows a typical course of an aortic aneurysm and its most serious life-threatening complication - aortic dissection. Aortic aneurysm is usually an asymptomatic disease (only $5-10 \%$ of patients have any symptoms and the symptoms are unfortunately nonspecific) until its catastrophic manifestation, AD, occurs. Aortic dissection claims high mortality. Many patients die prior to hospitalization, often without diagnosis. The results of the emer- gency aortic surgery remain unsatisfactory with in-hospital mortality reaching $30 \%$ despite the improvement in surgical procedure even in experienced centers [44]. We also know that elective aortic surgery is connected with much lower perioperative mortality, 3.7-8.3\% [45]. As AD tends to occur in an aneurysmal aorta, treatment of aneurysms before dissection occurs is essential for long-term survival. Current guidelines propose elective aortic surgery when the aortic size reaches $5.0 \mathrm{~cm}$ in case of MFS, BAV or other genetic syndromes, and $5.5 \mathrm{~cm}$ in other cases [7].

As has been pointed out, due to his refusal to undergo elective surgery, the patient was finally operated on in an emergency situation. The case report supports the need for early diagnosis and adequate management of thoracic aortic aneurysms, including an appropriate qualification for prophylactic aortic surgery, as such a procedure may reduce the prevalence of acute aortic syndromes and mortality rate resulting from $A A D$.

\section{References}

1. Braverman AC, Guven H, Beardslee MA, et al. The bicuspid aortic valve. Curr Probl Cardiol 2005; 30: 470-522.

2. Roos-Hesselink JW, Scholzel BE, Heijdra RJ, et al. Aortic valve and aortic arch pathology after coarctation repair. Heart 2003; 89: 1074-1077.

3. Siu SC, Silversides CK. Bicuspid aortic valve disease. J Am Coll Cardiol 2010; 55: 2789-2800.

4. Svensson LG, Blackstone EH, Cosgrove DM 3rd. Surgical options in young adults with aortic valve disease. Curr Probl Cardiol 2003; 28: 417-480.

5. Cecconi M, Nistri S, Quarti A, et al. Aortic dilatation in patients with bicuspid aortic valve. J Cardiovasc Med (Hagerstown) 2006; 7: 11-20.

6. Loscalzo ML, Goh DL, Loeys B, et al. Familial thoracic aortic dilation and bicommissural aortic valve: a prospective analysis of natural history and inheritance. Am J Med Genet A 2007; 143A: 1960-1967.

7. Hiratzka LF, Bakris GL, Beckman JA, et al. 2010 ACCF/AHA/AATS/ ACR/ASA/SCA/SCAI/SIR/STS/SVM Guidelines for the diagnosis and management of patients with thoracic aortic disease. A Report of the American College of Cardiology Foundation/American Heart Association Task Force on Practice Guidelines, American Association for Thoracic Surgery, American College of Radiology, American Stroke Association, Society of Cardiovascular Anesthesiologists, Society for Cardiovascular Angiography and Interventions, Society of Interventional Radiology, Society of Thoracic Surgeons, and Society for Vascular Medicine. J Am Coll Cardiol 2010; 55: e27-e129.

8. Hagan PG, Nienaber CA, Isselbacher EM, et al. The International Registry of Acute Aortic Dissection (IRAD): new insights into an old disease. JAMA 2000; 283: 897-903.

9. Januzzi JL, Isselbacher EM, Fattori R, et al. Characterizing the young patient with aortic dissection: results from the International Registry of Aortic Dissection (IRAD). J Am Coll Cardiol 2004; 43: 665-669.

10. Albornoz G, Coady MA, Roberts M, et al. Familial thoracic aortic aneurysms and dissections: incidence, modes of inheritance, and phenotypic patterns. Ann Thorac Surg 2006; 82: 1400-1405.

11. Pachulski RT, Weinberg AL, Chan KL. Aortic aneurysm in patients with functionally normal or minimally stenotic bicuspid aortic valve. Am J Cardiol 1991; 67: 781-782. 
12. Nistri S, Sorbo MD, Marin M, et al. Aortic root dilatation in young men with normally functioning bicuspid aortic valves. Heart 1999; 82: 19-22.

13. Cecconi M, Manfrin M, Moraca A, et al. Aortic dimensions in patients with bicuspid aortic valve without significant valve dysfunction. Am J Cardiol 2005; 95: 292-294.

14. de Sa M, Moshkovitz Y, Butany J, et al. Histologic abnormalities of the ascending aorta and pulmonary trunk in patients with bicuspid aortic valve disease: clinical relevance to the ross procedure. J Thorac Cardiovasc Surg 1999; 118: 588-594.

15. Fedak PW, de Sa MP, Verma S, et al. Vascular matrix remodeling in patients with bicuspid aortic valve malformations: implications for aortic dilatation. J Thorac Cardiovasc Surg 2003; 126: 797-806.

16. Fedak PW, Verma S, David TE, et al. Clinical and pathophysiological implications of a bicuspid aortic valve. Circulation 2002; 106 900-904.

17. Boyum J, Fellinger EK, Schmoker JD, et al. Matrix metalloproteinase activity in thoracic aortic aneurysms associated with bicuspid and tricuspid aortic valves. J Thorac Cardiovasc Surg 2004; 127 686-691.

18. Ikonomidis JS, Jones JA, Barbour JR, et al. Expression of matrix metalloproteinases and endogenous inhibitors within ascending aortic aneurysms of patients with bicuspid or tricuspid aortic valves. J Thorac Cardiovasc Surg 2007; 133: 1028-1036.

19. Klompas M. Does this patient have an acute thoracic aortic dissection? JAMA 2002; 287: 2262-2272.

20. Rogers AM, Hermann LK, Booher AM, et al. Sensitivity of the aortic dissection detection risk score, a novel guideline-based tool for identification of acute aortic dissection at initial presentation: results from the international registry of acute aortic dissection. Circulation 2011; 123: 2213-2218.

21. Hansen MS, Nogareda GJ, Hutchison SJ. Frequency of and inappropriate treatment of misdiagnosis of acute aortic dissection. Am J Cardiol 2007; 99: 852-856.

22. Sullivan PR, Wolfson AB, Leckey RD, et al. Diagnosis of acute thoracic aortic dissection in the emergency department. Am J Emerg Med 2000; 18: 46-50.

23. Raghupathy A, Nienaber CA, Harris KM, et al. Geographic differences in clinical presentation, treatment, and outcomes in type A acute aortic dissection (from the International Registry of Acute Aortic Dissection). Am J Cardiol 2008; 102: 1562-1566.

24. Kurabayashi M, Miwa N, Ueshima D, et al. Factors leading to failure to diagnose acute aortic dissection in the emergency room. J Cardiol 2011; 58: 287-293.

25. Torbicki A, Perrier A, Konstantinides S, et al. Guidelines on the diagnosis and management of acute pulmonary embolism: the Task Force for the Diagnosis and Management of Acute Pulmonary Embolism of the European Society of Cardiology (ESC). Eur Heart J 2008; 29: 2276-2315.

26. Meredith EL, Masani ND. Echocardiography in the emergency assessment of acute aortic syndromes. Eur J Echocardiogr 2009; 10: i31-i39.

27. Shiga T, Wajima Z, Apfel CC, et al. Diagnostic accuracy of transesophageal echocardiography, helical computed tomography, and magnetic resonance imaging for suspected thoracic aortic dissection: systematic review and meta-analysis. Arch Intern Med 2006; 166: 1350-1356.

28. Nienaber CA, von Kodolitsch Y, Nicolas V, et al. The diagnosis of thoracic aortic dissection by noninvasive imaging procedures. N Engl J Med 1993; 328: 1-9.
29. Borner N, Erbel R, Braun B, et al. Diagnosis of aortic dissection by transesophageal echocardiography. Am J Cardiol 1984; 54: 1157-1158.

30. Zeman RK, Berman PM, Silverman PM, et al. Diagnosis of aortic dissection: value of helical CT with multiplanar reformation and three-dimensional rendering. AJR Am J Roentgenol 1995; 164: 1375-1380.

31. Sommer T, Fehske W, Holzknecht N, et al. Aortic dissection: a comparative study of diagnosis with spiral CT, multiplanartransesophageal echocardiography, and MR imaging. Radiology 1996; 199: 347-352.

32. McMahon MA, Squirrell CA. Multidetector CT of aortic dissection: a pictorial review. Radiographics 2010; 30: 445-460.

33. Halpern EJ. Triple-rule-out CT angiography for evaluation of acute chest pain and possible acute coronary syndrome. Radiology 2009; 252: 332-345.

34. Frauenfelder T, Appenzeller P, Karlo C, et al. Triple rule-out CT in the emergency department: protocols and spectrum of imaging findings. Eur Radiol 2009; 19: 789-799.

35. Becker HC, Johnson T. Cardiac CT for the assessment of chest pain: Imaging techniques and clinical results. Eur J Radiol 2012; 81: 3675-3679.

36. Sarasin FP, Louis-Simonet M, Gaspoz JM, et al. Detecting acute thoracic aortic dissection in the emergency department: time constraints and choice of the optimal diagnostic test. Ann Emerg Med 1996; 28: 278-288.

37. Erbel R, Alfonso F, Boileau C, et al. Diagnosis and management of aortic dissection. Eur Heart J 2001; 22: 1642-1681.

38. Wei H, Schiele F, Meneveau N, et al. The value of intravascular ultrasound imaging in diagnosis of aortic penetrating atherosclerotic ulcer. Eurolntervention 2006; 1: 432-437.

39. Fan QK, Wang WW, Zhang ZL, et al. Evaluation of D-dimer in the diagnosis of suspected aortic dissection. ClinChem Lab Med 2010; 48: 1733-1737.

40. Shimony A, Filion KB, Mottillo S, et al. Meta-analysis of usefulness of D-dimer to diagnose acute aortic dissection. Am J Cardiol 2011; 107: 1227-1234.

41. Suzuki T, Distante A, Eagle K. Biomarker-assisted diagnosis of acute aortic dissection: how far we have come and what to expect. Curr Opin Cardiol 2010; 25: 541-545.

42. Trimarchi S, Sangiorgi G, Sang X, et al. In search of blood tests for thoracic aortic diseases. Ann Thorac Surg 2010; 90: 1735-1742.

43. Anagnostopoulos CE, Prabhakar MJ, Kittle CF. Aortic dissections and dissecting aneurysms. Am J Cardiol 1972; 30: 263-273.

44. Trimarchi S, Nienaber CA, Rampoldi V, et al. Contemporary results of surgery in acute type $A$ aortic dissection: The International Registry of Acute Aortic Dissection experience. J Thorac Cardiovasc Surg 2005; 129: 112-122.

45. Gazoni LM, Speir AM, Kron IL, et al. Elective thoracic aortic aneurysm surgery: better outcomes from high-volume centers. J Am Coll Surg 2010; 210: 855-860. 